\title{
Consumo máximo de oxigênio e limiar anaeróbio de jogadores de futebol: comparação entre as diferentes posições
}

\author{
Pedro Balikian ${ }^{1}$, Aparecido Lourenção ${ }^{2}$, Luiz Fernando Paulino Ribeiro ${ }^{3}$, \\ Wilham Tadeu Lara Festuccia ${ }^{4}$ e Cassiano Merussi Neiva ${ }^{5}$
}

\section{RESUMO}

O grau de desenvolvimento das capacidades físicas no futebol é fator determinante do nível desportivo do jogador. O objetivo do presente estudo foi comparar valores de limiar anaeróbio e consumo máximo de oxigênio entre jogadores profissionais de futebol de diferentes posições. Para tanto, 25 atletas (idade $=22,08 \pm 8,28$ anos, peso $=76,12 \pm$ $9,8 \mathrm{~kg}$, altura $=179,8 \pm 7,1 \mathrm{~cm}$ e relação corporal $=12,21 \pm$ $3,67 \%$ de gordura corporal) foram divididos em cinco grupos, como se segue: goleiros (GO), zagueiros (ZA), laterais (LA), meio-campistas (MC) e atacantes (AT). O $\mathrm{VO}_{2}$ max foi determinado em esteira ergométrica através de análise direta e a velocidade de corrida correspondente ao limiar anaeróbio fixo de $4 \mathrm{mM}(\mathrm{V} 4 \mathrm{mM})$, em teste de campo ( $2 \mathrm{x}$ $1.000 \mathrm{~m}$ a 90 e $95 \%$ da velocidade máxima para a distância) através de interpolação linear.

1. Pró-Reitoria de Pesquisa e Pós-Graduação - Unoeste - Presidente Prudente, SP.

2. Faculdade de Ciências e Tecnologia - Unesp - Presidente Prudente, SP.

3. Departamento de Ciências Fisiológicas - UFSCar - São Carlos, SP.

4. Departamento de Fisiologia e Bioquímica - USP - Ribeirão Preto, SP.

5. Faculdade de Ciências - Unesp - Bauru, SP.

Recebido em 16/12/01

Segunda versão recebida em 1/4/02

Aceito em 14/4/02

Endereço para correspondência:

Pedro Balikian

Lafine - Laboratório de Fisiologia e Nutrologia Experimentais em Exercício Universidade de Ribeirão Preto

Av. Costábile Romano, 2.201 - Sala T 36 - Núcleo Multiprofissional

14096-380 - Ribeirão Preto, SP

Tel.: (16) 603-6772

E-mail: pjunior@posgrad.unoeste.br

\begin{tabular}{|c|c|c|c|c|c|}
\hline & $\mathrm{GO}(\mathrm{n}=5)$ & $Z A(n=5)$ & $L A(n=5)$ & $M C(n=5)$ & AT $(n=5)$ \\
\hline $\begin{array}{l}\text { V4mM } \\
\left(\mathrm{km} \cdot \mathrm{h}^{-1}\right)\end{array}$ & $12,66 \pm 0,89 *$ & $13,15 \pm 1,56$ & $14,33 \pm 0,66^{\#}$ & $14,11 \pm 0,51^{\#}$ & $13,23 \pm 0,86$ \\
\hline $\begin{array}{c}\dot{\mathrm{V}} \mathrm{O}_{2} \max \\
\left(\mathrm{ml} \cdot \mathrm{kg} \cdot \mathrm{min}^{-1}\right)\end{array}$ & $52,68 \pm 3,21 *$ & $60,28 \pm 6,23$ & $61,12 \pm 5,33$ & $61,01 \pm 7,14$ & $59,94 \pm 6,19$ \\
\hline
\end{tabular}

* $p<0,05$ em relação às outras posições. * $p<0,05$ em relação à ZA e AT.

A V4mM foi menor $(\mathrm{p}<0,05)$ para o grupo GO em relação aos demais grupos. Além disso, os grupos LA e MC apresentaram valores de $\mathrm{V} 4 \mathrm{mM}$ significantemente maiores em relação aos grupos ZA e AT. O grupo GO mostrou $\dot{\mathrm{V}}_{2}$ max significantemente menor em relação a todos os outros grupos, sendo que estes últimos não apresentaram diferença entre si. Uma vez que os atletas de diferentes posições não realizavam treinamento diferenciado, os autores creditam as diferenças encontradas à especificidade da movimentação durante partidas e coletivos.

Palavras-chave: Futebol. Limiar anaeróbio. Potência aeróbia.

\section{ABSTRACT}

Maximal oxygen uptake and anaerobic threshold in professional soccer players: comparison between different positions

The development of physical capacities in soccer determines the player's level of performance. The aim of this study was to compare anaerobic threshold and maximal oxygen uptake values between professional soccer players playing in different positions. Twenty-five male athletes (age $=22.08 \pm 8.82$ years, weight $=76.12 \pm 9.8 \mathrm{~kg}$, height $=$ $179.8 \pm 7.1 \mathrm{~cm}$ and body composition $=12.21 \pm 3.67 \%$ body fat) were divided into five groups as follows: goalkeepers (GO), full backs (FB), sideways (SW), midfield (MF) and forwards $(\mathrm{FO})$ and evaluated twice in two to three days period. For direct maximal oxygen uptake ( $\dot{V}_{2}$ max) determination, subjects were submitted to an incremental continuous treadmill exercise until fatigue occurred. Two- 
speeds track test $(2 \times 1,000 \mathrm{~m}$ at 90 and $95 \%$ of maximal speed) and linear interpolation were employed for $4 \mathrm{mM}$ blood lactate running speed (V4mM) or anaerobic threshold assessment. Main results are present below.

\begin{tabular}{|c|c|c|c|c|c|}
\hline & $\mathrm{GO}(\mathrm{n}=5)$ & $\mathrm{FB}(\mathrm{n}=5)$ & $S W(n=5)$ & MF $(n=5)$ & FO (n = 5) \\
\hline $\begin{array}{l}\mathrm{V} 4 \mathrm{mM} \\
\left(\mathrm{km} \cdot \mathrm{h}^{-1}\right)\end{array}$ & $12.66 \pm 0.89 *$ & $13.15 \pm 1.56$ & $14.33 \pm 0.66^{\#}$ & 14.11 & $13.23 \pm 0.86$ \\
\hline $\begin{array}{c}\dot{\mathrm{VO}_{2} \max } \\
\left(\mathrm{ml} . \mathrm{kg} \cdot \mathrm{min}^{-1}\right)\end{array}$ & $52.68 \pm 3.21^{*}$ & $60.28 \pm 6.23$ & $61.12 \pm 5.33$ & $61.01 \pm 7.14$ & $59.94 \pm 6.19$ \\
\hline
\end{tabular}

${ }^{*} \mathrm{p}<0.05$ as compared to other positions; ${ }^{\mathrm{p}} \mathrm{p}<0.05$ as compared to FB and FO.

V4mM for GO group was significantly lower ( $p<0.05$ ) than those found for any other group. In addition, $S W$ and $M F$ presented V4mM values which were significantly greater $(p<0.05)$ than those for $\mathrm{FB}$ and $\mathrm{FO}$. $\mathrm{VO}_{2}$ max for GO was significantly lower $(p<0.05)$ than those for other groups, which were not significantly different $(p>0.05)$ from one another. Since these athletes were under the same training program, the differences above may be explained by different metabolic solicitation (position specificity) during official and training games.

Key words: Soccer. Anaerobic threshold. Aerobic power.

\section{INTRODUÇÃO}

O futebol de campo é uma atividade complexa, que exige do jogador o desenvolvimento de diversas capacidades físicas, motoras e psíquicas. O grau de desenvolvimento das capacidades físicas no futebol é fator determinante do nível desportivo do jogador. Por esse motivo, essas aptidões também devem ser trabalhadas fora do campo de jogo ${ }^{1,2}$.

Diferentes estudos têm mostrado que o deslocamento dos jogadores durante as partidas é determinado principalmente pela posição ou função tática exercida ${ }^{2-4}$. Além disso, o nível do campeonato exerce influência na distância total percorrida durante o jogo ${ }^{2,5}$. Estes dados sugerem que, dependendo da função tática que exerce no time, cada jogador tem um nível de solicitação metabólica, que por sua vez exige e gera adaptações diferenciadas nos processos de produção de energia ${ }^{1}$.

Apesar da vasta literatura a respeito das características fisiológicas de desportistas de diferentes modalidades ${ }^{6-10}$, poucos trabalhos analisaram a influência de diferentes funções táticas sobre as características fisiológicas de futebolistas ${ }^{1,2,11-14}$ Assim, o presente estudo teve como objetivo comparar jogadores de futebol de diferentes posições no que diz respeito ao consumo máximo de oxigênio ( $\mathrm{V}_{2} \max$ ) e limiar anaeróbio.

\section{MÉTODOS}

\section{Voluntários}

Foram voluntários deste estudo 25 jogadores profissionais de futebol de campo que atuavam na segunda divisão do campeonato paulista. As características dos atletas são apresentadas na tabela 1. Os voluntários foram subdivididos em cinco grupos de cinco jogadores, como segue: goleiros (GO), zagueiros (ZA), laterais (LA), meio-campistas (MC) e atacantes (AT). Após a aprovação do estudo pelo Conselho de Ética, os atletas foram informados sobre a natureza e metodologia do experimento, e assinaram termo de consentimento esclarecido. Foram, então, submetidos de forma randômica a duas avaliações, separadas entre si por um período de dois a três dias.

\begin{tabular}{|c|c|c|c|c|}
\hline \multicolumn{5}{|c|}{$\begin{array}{c}\text { TABELA } 1 \\
\text { Média }(\bar{X}) \text { e desvio padrão (DP) das características } \\
\text { antropométricas dos voluntários estudados }(n=25)\end{array}$} \\
\hline & $\begin{array}{l}\text { Idade } \\
\text { (anos) }\end{array}$ & $\begin{array}{c}\text { Peso } \\
\text { (kg) }\end{array}$ & $\begin{array}{c}\text { Altura } \\
\text { (cm) }\end{array}$ & $\begin{array}{c}\text { Gordura } \\
\text { corporal (\%) }\end{array}$ \\
\hline $\bar{x}$ & 22,08 & 76,12 & 179 & 12,21 \\
\hline DP & 8,28 & 9,8 & 7 & 3,67 \\
\hline
\end{tabular}

\section{Protocolo experimental}

Determinação do consumo máximo de oxigênio: Para a determinação do $\dot{\mathrm{V}}_{2}$ max, os sujeitos realizaram uma sessão de exercício contínuo e progressivo até exaustão em esteira ergométrica modelo ATL 10200 (Inbramed $^{\circledR}$ ). A velocidade inicial foi de $10,0 \mathrm{~km} / \mathrm{h}$, com incrementos de $1,0 \mathrm{~km} / \mathrm{h}$ a cada minuto, sendo a elevação fixada em $1 \%$.

$\mathrm{O} \mathrm{VO}_{2}$ foi mensurado continuamente durante todo o protocolo de avaliação, a partir do gás expirado, utilizando para isto o sistema Vista $C P X^{\circledR}$, Vacumed, 1996. Este sistema permitiu a dosagem do $\mathrm{O}_{2}$ e $\mathrm{CO}_{2}$ do ar expirado a cada 30s, utilizando-se da câmara de mistura e analisadores Oxygen Analyser OM-11 e Carbon Dioxide analiser LB-2, respectivamente, e a determinação do volume de ar expirado por meio de fluxômetro $K-520$. Os sinais dos equipamentos foram devidamente processados e analisados por meio de software do sistema Vista CPX, fornecendo os valores de consumo de oxigênio $\left(\dot{\mathrm{VO}}_{2}\right)$, produção de $\mathrm{CO}_{2}$, razão de trocas respiratórias $(\mathrm{R})$ e ventilação pulmonar (VE btps) a cada 30 segundos.

Determinação do limiar anaeróbio: Para a determinação da velocidade de corrida correspondente ao limiar anaeróbio de concentração fixa $\left(\mathrm{V}_{4 \mathrm{mM}}\right)$, foi utilizado teste de campo seguindo-se protocolo similar ao proposto por Mader et al..$^{15}$. Os sujeitos correram $2 \times 1.000 \mathrm{~m}$, respectivamente, a 90 e $95 \%$ da velocidade máxima para o percurso 
(previamente determinado), com 20min de pausa entre os tiros ${ }^{9}$. A velocidade foi controlada a cada 100 metros por apito e sinal visual.

Nos minutos 1, 3 e 5 após cada tiro foram coletados 25 microlitros $(\mu \mathrm{l})$ de sangue do lóbulo da orelha em capilares heparinizados, armazenados em microtubos de polietileno com tampa, tipo ependorfs, com $50 \mu \mathrm{l}$ de $\mathrm{NaF}$ a $1 \%$, sendo que as amostras foram congeladas até a leitura para aferição do lactato sanguíneo através de um analisador eletroquímico YSL 2700-STAT (Yellow Springs Co., EUA). Para a determinação da $\mathrm{V}_{4 \mathrm{mM}}$ foi considerada apenas a mais alta concentração de lactato após cada tiro. Desse modo, para cada tiro foi determinada a velocidade média e sua respectiva concentração de lactato e, por interpolação linear, foi calculada a velocidade correspondente ao limiar anaeróbio $\left(\mathrm{V}_{4 \mathrm{mM}}\right)$ para concentração determinada de $4 \mathrm{mM}$ de lactato.

\section{Análise estatística}

A média aritmética e o desvio padrão foram calculados para todas as variáveis estudadas. Para as comparações entre grupos foi utilizada a ANOVA, com posterior aplicação de teste complementar de Bonferroni t-teste. Para todas as análises foi adotado o coeficiente de significância estatística $\mathrm{p}<0,05$.

\section{RESULTADOS}

A $\mathrm{V}_{4 \mathrm{mM}}$ foi menor $(\mathrm{p}<0,05)$ para o grupo $\mathrm{GO}(12,66 \pm$ $\left.0,89 \mathrm{~km} \cdot \mathrm{h}^{-1}\right)$ em relação aos demais grupos. Além disso, os grupos LA $\left(14,33 \pm 0,66 \mathrm{~km} \cdot \mathrm{h}^{-1}\right)$ e MC $\left(14,11 \pm 0,51 \mathrm{~km} \cdot \mathrm{h}^{-1}\right)$ apresentaram valores de $\mathrm{V}_{4 \mathrm{mM}}$ maiores $(\mathrm{p}<0,05)$ em relação aos grupos ZA $\left(13,15 \pm 1,56 \mathrm{~km} \cdot \mathrm{h}^{-1}\right)$ e AT $(13,23 \pm$ $0,86 \mathrm{~km} \cdot \mathrm{h}^{-1}$ ) (figura 1 ).

O grupo GO também apresentou $\mathrm{V}_{2} \max (52,68 \pm$ $\left.3,21 \mathrm{ml} \cdot \mathrm{kg} \cdot \mathrm{min}^{-1}\right)$ inferior $(\mathrm{p}<0,05)$ em relação a todos os outros grupos $(\mathrm{ZA}=60,28 \pm 6,23 ; \mathrm{LA}=61,12 \pm 5,33 ; \mathrm{MC}=$ $61,01 \pm 7,14$ e AT $\left.=59,94 \pm 6,19 \mathrm{ml} \cdot \mathrm{kg} \cdot \mathrm{min}^{-1}\right)$, sendo que estes não apresentaram diferença $(p>0,05)$ entre si (figura 2).

\section{DISCUSSÃO}

O futebol de campo é uma modalidade esportiva coletiva e complexa, sendo que as diversas posições ou funções táticas exercidas determinam grande variabilidade individual no que diz respeito à intensidade e volume dos deslocamentos em partida e, conseqüentemente, às respostas fisiológicas frente ao jogo.

A caracterização funcional de atletas de diferentes modalidades esportivas é alvo de grande número de estudos na fisiologia do exercício. De modo geral, atletas especia-

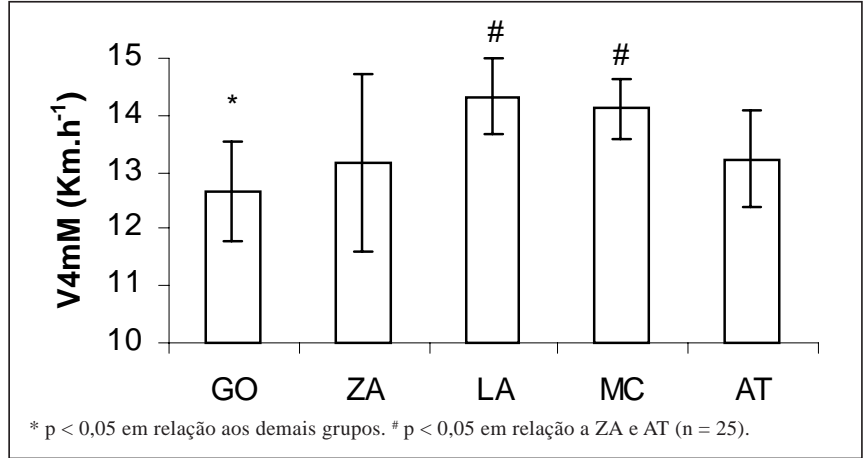

Fig. 1 - Comparação entre o limiar anaeróbio $(v 4 m M)$ de jogadores de diferentes posições. $G O=$ goleiros; $Z A=$ zagueiros; $L A=$ laterais; $M C$ = meio-campistas; e AT = atacantes .

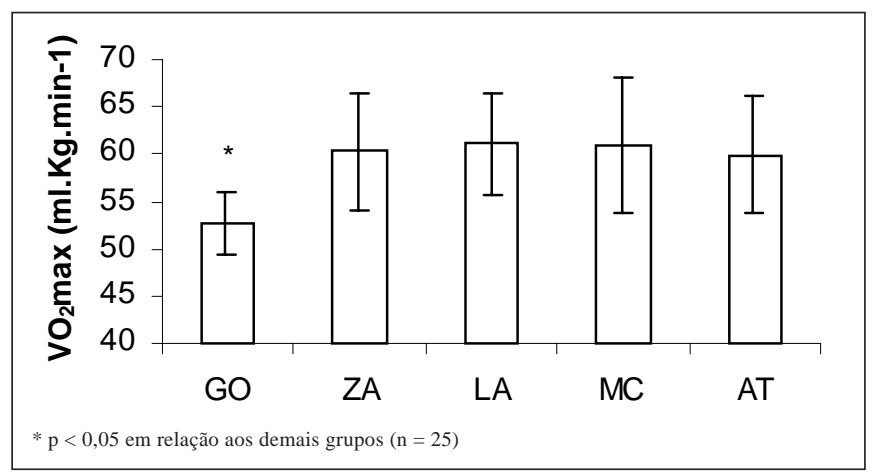

Fig. 2 - Comparação entre o consumo máximo de oxigênio ( $\dot{\mathrm{V} O}, \max )$ de jogadores de diferentes posições. $G O=$ goleiros; $Z A=$ zagueiros; $L A=$ laterais $; M C=$ meio-campistas $;$ e $A T=$ atacantes .

listas em eventos de longa duração apresentam altos níveis de capacidade aeróbia e limiar anaeróbio, indicando grande desenvolvimento do sistema cardiorrespiratório e alta capacidade oxidativa do tecido muscular ${ }^{16}$. Por outro lado, atletas cujas atividades envolvem predominantemente força e potência apresentam grande desenvolvimento das vias anaeróbias (lática e/ou alática) de produção de energia ${ }^{17}$.

No que diz respeito ao futebol de campo, não só o esquema tático e posicionamento dos jogadores, mas também a infinidade de situações de jogo, tornam difícil a quantificação da importância de cada via energética durante a realização de uma partida. Um importante passo na otimização da dinâmica de jogo nesse esporte, do ponto de vista fisiológico, é o estudo comparativo da capacidade funcional de jogadores de diferentes posições, com o intuito não só de compreender a solicitação metabólica das diferentes funções, mas também de subsidiar a elaboração de treinamentos específicos.

Existem evidências de que o treinamento em futebol não apresenta a especificidade necessária para as diferentes funções táticas do $\operatorname{jog}_{0}{ }^{18}$. Sustentando tal afirmação está o 
fato de que treinamentos diferenciados para cada posição, exceto para os goleiros, não eram adotados no clube ao qual pertenciam os atletas do presente estudo, sendo que as diferenças encontradas têm como causa mais provável a sobrecarga proporcionada pelas partidas e treinamentos coletivos.

As velocidades referentes ao limiar anaeróbio $\left(\mathrm{V}_{4 \mathrm{mM}}\right)$ para as diferentes posições, verificadas neste trabalho, foram ligeiramente inferiores às encontradas em um estudo ${ }^{18}$ envolvendo 51 jogadores profissionais, que determinou para goleiros, laterais, zagueiros, meio-campistas e atacantes $\mathrm{V}_{4 \mathrm{mM}}$ de, respectivamente, $13,6 \pm 0,7 ; 14,9 \pm 0,9 ; 14,6 \pm$ 1,$1 ; 15,4 \pm 0,9$ e $15 \pm 0,8 \mathrm{~km} \cdot \mathrm{h}^{-1}$. Além disso, a única diferença verificada pelos autores acima se deu entre goleiros e meio-campistas, não sustentando a hipótese de que a exigência metabólica em partida gere adaptações mensuráveis entre as demais posições.

Contrariando esses dados, em nosso experimento jogadores de meio-campo e laterais apresentaram $\mathrm{V}_{4 \mathrm{mM}}$ maiores em relação aos demais grupos. Tais resultados podem ser parcialmente explicados com base nos dados de estudos que, comparando o deslocamento de jogadores em partida, verificaram que meio-campistas e laterais percorriam distâncias cerca de 5\% maiores que atacantes e zaguei$\operatorname{ros}^{2,3,11}$. Dessa forma, inferimos que o maior volume de corrida em jogos oficiais e coletivos pode resultar em adaptações funcionais que se expressam em valores diferenciados de limiar anaeróbio.

O maior deslocamento de meio-campistas e laterais em partida pode ser explicado pelas funções táticas exercidas por estes atletas no futebol moderno, ou seja, tais jogadores têm como função tanto o ataque (armação e finalização de jogadas) como a defesa (marcação aos adversários), ocasionando movimentação ampla e constante pelo campo de jogo $0^{1,11,19}$

Por outro lado, os valores menores de $\mathrm{V}_{4 \mathrm{mM}}$ para os grupos de zagueiros e atacantes podem ser explicados por ambas as posições terem funções táticas relacionadas a um setor restrito do campo, o setor defensivo para os zagueiros que desempenham marcação e/ou cobertura dos laterais e meio-campistas, e o setor ofensivo para os atacantes, cujas obrigações incluem armação, finalização e marcação da saída de bola próximo à meta adversária ${ }^{11,19}$.

Apresentando limiar anaeróbio menor entre todos os grupos estudados encontram-se os goleiros que, além de realizar treinamento diferenciado para a posição, apresentam movimentação específica em partida. Este grupo de jogadores realiza exclusivamente movimentos de curta duração e alta intensidade (defesas em chutes e cabeçadas a gol, saídas da meta para interceptação de lançamentos, cruzamentos e reposições de bola em jogo), dependendo pre- dominantemente do sistema anaeróbio alático para a produção de energia ou ATP-CP ${ }^{20}$. Os goleiros apresentam, quase sempre, recuperação passiva e também baixo volume de deslocamento quando comparados com outras posições ${ }^{21}$.

No que diz respeito ao $\mathrm{V}_{2} \max$, quando os jogadores são dispostos num só grupo, os valores encontrados neste estudo são próximos aos relatados pela literatura $(62,0 \pm$ $\left.4,7 \mathrm{ml} \cdot \mathrm{kg}^{-1} \cdot \mathrm{min}^{-1}\right)^{22} ;\left(60,0-65,0 \mathrm{ml} \cdot \mathrm{kg}^{-1} \cdot \mathrm{min}^{-1}\right) ;(55,0-$ $\left.65,0 \mathrm{ml} \cdot \mathrm{kg}^{-1} \cdot \mathrm{min}^{-1}\right)^{23} ;\left(60,0 \pm 1,0 \mathrm{ml} \cdot \mathrm{kg}^{-1} \cdot \mathrm{min}^{-1}\right)^{24} ;(56,20 \pm$ $\left.6,23 \mathrm{ml} \cdot \mathrm{kg}^{-1} \cdot \mathrm{min}^{-1}\right)^{21}$ e $\left(58,7 \pm 4,1 \mathrm{ml} \cdot \mathrm{kg}^{-1} \cdot \mathrm{min}^{-1}\right)^{25}$. Isso implica uma semelhança bastante acentuada dos resultados referidos na literatura com os aqui apresentados.

Quando considerados os diferentes grupos, os valores referentes ao $\dot{\mathrm{V}}_{2}$ max obtidos em nosso estudo apresentam-se semelhantes para os goleiros $\left(54,01 \mathrm{ml} \cdot \mathrm{kg}^{-1} \cdot \mathrm{min}^{-1}\right) \mathrm{e}$ discretamente superiores para zagueiros, meio-campistas, atacantes e laterais $\left(55,49 ; 55,90 ; 56,07 ; 59,90 \mathrm{ml} \cdot \mathrm{kg}^{-1} \cdot \mathrm{min}^{-1}\right.$, respectivamente) ${ }^{21}$. Já quando comparados com os valores obtidos indiretamente para goleiros, laterais, zagueiros e meio-campistas $\left(60,19 ; 65,38 ; 61,85 ; 64,53 \mathrm{ml} . \mathrm{kg} \cdot \mathrm{min}^{-1}\right.$, respectivamente) ${ }^{26}$, os valores que encontramos apresentam-se ligeiramente inferiores para goleiros, zagueiros e meio-campistas, sendo semelhantes para o grupo de laterais.

Nossos dados apontam diferença apenas entre goleiros e os demais grupos, concordando com dados apresentados pela literatura ${ }^{21}$. Entretanto, através de metodologia indireta para a determinação do $\dot{\mathrm{V}}_{2}$ max de jogadores da categoria juniores, verificou-se que meio-campistas e laterais apresentaram valores superiores aos dos goleiros, zagueiros e atacantes ${ }^{26}$. Essas diferenças apresentadas pela análise indireta do $\mathrm{V}_{2}$ max entre as diversas posições podem estar relacionadas à metodologia utilizada e à faixa etária dos atletas.

Para indivíduos saudáveis, mais de $90 \%$ da variabilidade do $\dot{\mathrm{V}}_{2} \max$ são determinados geneticamente ${ }^{27}$. Sendo assim, essa variável pode apresentar limitações para avaliar possíveis diferenças de capacidade aeróbia entre atletas, pois, muitas vezes, um considerável ganho no condicionamento aeróbio pode não implicar uma relação direta com aumento do $\mathrm{VO}_{2}$ max e, sim, ser refletido de forma mais clara em outras variáveis bioquímicas do desempenho motor.

Além disso, a maior sensibilidade de variáveis lactacidêmicas (como o limiar anaeróbio) em relação ao $\mathrm{V}_{2} \max$ em discriminar a capacidade de realização de trabalho aeróbio talvez seja explicada pelo fato de essas variáveis serem determinadas por diferentes fatores. Apesar de alguma controvérsia ${ }^{28}$, o consumo máximo de oxigênio parece ser limitado por fatores centrais ou cardiovasculares, como débito cardíaco e volume de ejeção, enquanto o limiar de 
lactato está relacionado a fatores periféricos, como a capacidade respiratória muscular ${ }^{29}$, densidade mitocondrial e tipo de fibra muscular predominante ${ }^{30,31}$.

Dessa forma, a análise lactacidêmica durante o exercício incremental é, nos dias atuais, a mais importante avaliação relacionada ao diagnóstico da capacidade de realização de trabalho aeróbio em pacientes ${ }^{32}$ e atletas ${ }^{33}$.

Apesar de a metodologia adotada neste estudo para a identificação do limiar anaeróbio apresentar limitações relacionadas à utilização de valor lactacidêmico fixo e à necessidade de extrapolação ou interpolação dos dados ${ }^{34,35}$, esta ainda é muito empregada no meio esportivo devido à sua praticidade, especialmente quando o número de atletas a ser avaliado num curto período de tempo é grande, como no caso de esportes coletivos como o futebol.

\section{CONCLUSÃO}

Com base nos dados obtidos, concluímos que jogadores de futebol de campo de variadas posições apresentam níveis diferenciados de condicionamento aeróbio, possivelmente devido às diversas sobrecargas metabólicas impostas durante partidas e treinamentos coletivos.

\section{REFERÊNCIAS}

1. Reilly T. Energetics of high-intensity exercise (soccer) with particular reference to fatigue. J Sports Sci 1997;15:257-63.

2. Rienzi E, Drust B, Reilly T, Carter JEL, Martin A. Investigation of anthropometric and work-rate profiles of elite South American international soccer players. J Sports Med Phys Fitness 2000;40:162-9.

3. Ekblon B. Applied physiology of soccer. Sports Med 1986;3:50-60.

4. Ohashi J, Togari H, Isokawa M. Measuring movement speeds and distance covered during soccer match-play. Science and Football. Proceedings of the First World Congress of Science and Football. London, New York. E. and S.F. Spon, 1988:329-34.

5. Withers RT, Maricic Z, Wasilewski S, Kelly L. Match analysis of Australian professional soccer players. J Hum Mov St 1982;8:159-76.

6. Ohkuwa T, Miyamura M. Peak blood lactate after $400 \mathrm{~m}$ sprinting in sprinters and long-distance runners. Jpn J Physiol 1984;34:552-6.

7. O'Toole ML, Hiller WD, Roaltad MS, Douglas PS. The relation of exercise test variables to bike performance times during the Hawaii Ironman triathlon. Med Sci Sports Exerc 1988;20:S-50.

8. Kokubun E, Daniel JF. Relações entre a intensidade e duração das atividades em partida de basquetebol com as capacidades aeróbicas e anaeróbicas: estudo pelo lactato sanguíneo. Rev Paul Educ Fís 1992;6:3746.

9. Denadai BS, Balikian Júnior P. Relação entre limiar anaeróbio e performance no Short Triathlon. Rev Paul Educ Fís 1995;9:10-15.

10. Balikian Júnior P, Denadai BS. Aplicações do limiar anaeróbio determinado em teste de campo para o ciclismo: comparação com valores obtidos em laboratório. Motriz 1996;2:26-31.

11. Van Gool D, van Gerven D, Boutmans J. The physiological load imposed on soccer players during real match-play. In: Science and Football Committee. World Congress of Science and Football. Liverpool, 1988:52-9.

12. Drust B, Reilly T, Rienzi E. Analysis of work rate in soccer. Sports Exer Inj 1998;4:151-5.
13. Tourny-Chollet, C, Leroy D, Leger H, Beuret-Blanquart F. Isokinetic knee muscle strength of soccer players according to their position. Isokinetics and exercise science 2000;8:187-93.

14. Al-Hazzaa HM, Almuzaini KS, Al-Rejaee SA, Sulaiman MA, Dafterdar MY, Alghadedi A, et al. Aerobic and anaerobic power characteristics of Saudi elite soccer players. J Sports Med Phys Fitness 2001;41:54-61.

15. Mader A, Heck H, Hollmann W. Evaluation of lactic acid anaerobic energy contribution by determination of post exercise lactic concentration of ear capillary blood in middle-distance runners and swimmers. Exerc Physiol 1978;4:187-94.

16. Williams, MH. Ergogenic Aids: a mean to Citius, Altius, Fortius, and Olympic Gold? Res Q Exerc Sport 1996;67:S58-64.

17. Greenhaff P. Creatine and its application as an ergogenic aid. Int J Sport Nutr 1995;5:S100-S110.

18. Santos JW, Kokubun E. Limiar anaeróbio de atletas profissionais de futebol nas diferentes posições de jogo. Motriz 1999;5:1.

19. Anjos R. Futebol - Regras, esquemas táticos, posições e funções: do goleiro ao ponta-esquerda. São Paulo: Rumo, 1990.

20. Cometti G, Maffiuletti NA, Pousson M, Chatard JC, Maffulli N. Isokinetic strength and anaerobic power of elite, subelite and amateur French soccer players. Int J Sports Med 2001;22:45-51.

21. Barros TL, Lotufo RF, Mine F. Consumo máximo de oxigênio em jogadores de futebol. Revista Brasileira de Atividade Física e Saúde 1996;1: 24-6.

22. Withers RT, Roberts RDD, Davies GJ. The maximum aerobic power, anaerobic power and body composition of South Australian male representatives in athletics, basketball, field hockey and soccer. J Sports Med 1977;1:391-400

23. Balson V. Handbook of sports medicine and science. Football (Soccer). $1^{\text {st }}$ ed. Oxford: Blackwell Scientific Publications, 1994.

24. Bangsbo J, Norregaard L, Thorso F. Activity profile of competition soccer. Can J Sports Sci 1991;16:110-6.

25. Rhodes EC, Mosher RE, Mckenzie DC, Franks IM, Potts JE, Wenger HA. Physiological profiles of the Canadian Olympic Soccer Team. Can J Appl Sports Sci 1986;1:31-6.

26. Gonçalves GA, Samulski D. Comparação do $\dot{V} \mathrm{O}_{2}$ max estimado, tempo de corrida de 50 metros e carga psíquica de jogadores de futebol de posições diferentes, de equipes da categoria júnior, da região metropolitana de Belo Horizonte - Minas Gerais. Revista Brasileira de Ciências do Esporte 1997;18:174-9.

27. Klissouras V. Hereditability of adaptative variation. J Appl Physiol 1971; 31:338-44.

28. Bergh U, Ekblom B, Astrand PO. Maximal oxygen uptake "classical" versus “contemporary" viewpoints. Med Sci Sports Exerc 2000;32:858.

29. Wasserman DH, Williams PE, Lacy DB, Goldstein RE, Cherrington AD. Exercise-induced fall in insulin and hepatic carbohydrate metabolism during muscular work. Am J Physiol 1989;256:E500-9.

30. Denis C, Fouquet R, Poty P, Geyssant A, Lacour JR. Effect of 40 weeks of endurance training on the anaerobic threshold. Int J Sports Med 1982; 3:208-14.

31. Hurley BF, Seals DR, Ehsani AA, Cartier LJ, Dalsky GP, Hagberg JM, et al. Effects of high-intensity strength training on cardiovascular function. Med Sci Sports Exerc 1984;16:483-8.

32. Coyle EF, Martin WH, Ehsani AA, Haberg JM, Bloomfield SA, Sinacore DR, et al. Blood lactate threshold in some well-trained ischemic heart disease patients. J Appl Physiol 1983;54: 18-23.

33. Weltman A. The blood lactate response to exercise. Champaign, IL: Human Kinetics, 1995.

34. Maassen N, Busse MW. The relationship between lactic acid and work load: a measure for endurance capacity or an indicator of carbohydrate deficiency? Eur J Appl Physiol 1989;58:728-37.

35. Reilly T, Woodbridge V. Effects of moderate dietary manipulations on swim performance and on blood lactate-swimming velocity curves. Int J Sports Med 1999;20:93-7. 